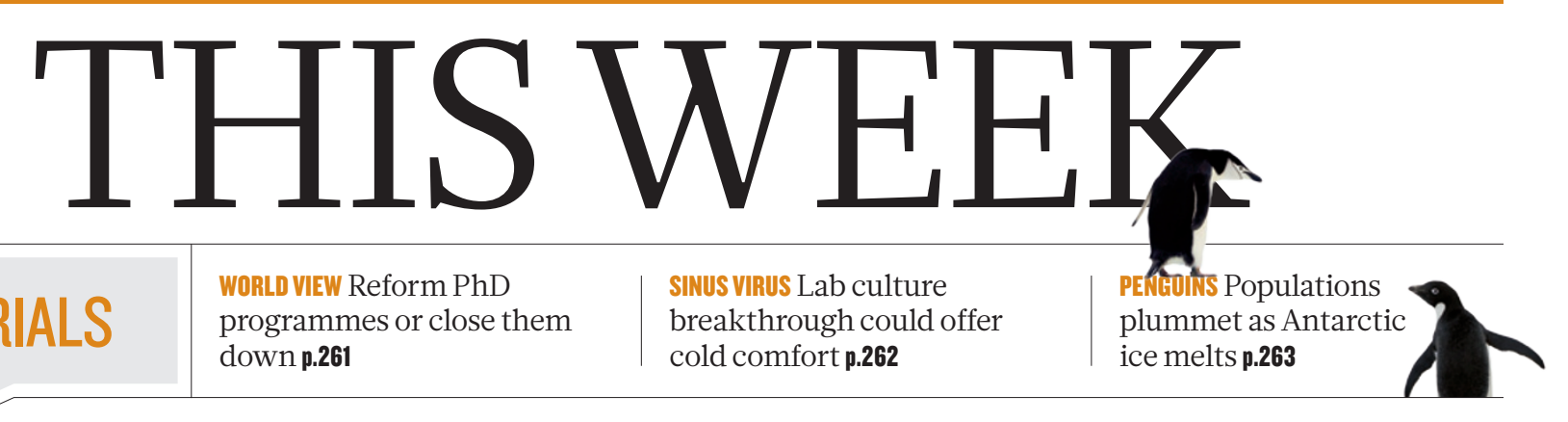

EDITORIALS programmes or close them down p.261

\title{
Accidents in waiting
}

\section{Every researcher and institution should question their own attitudes to safety in the lab after the death of an undergraduate student in a Yale University workshop.}

$\mathrm{T}$ The shocking death of physics and astronomy undergraduate student Michele Dufault in a machine shop at Yale University in New Haven, Connecticut, last week should grab the attention of researchers and safety officers at universities across the United States, and the wider world. Rightly, the immediate focus is on whether the university could have taken more precautions to prevent the accident. But whatever the verdict, Dufault's death — late at night and probably while working alone - should remind every researcher to consider their own attitude to safety, and whether it is crowded out by other priorities.

Most scientists are well aware of poor safety practices in their laboratories - such as too many people working on their own, students not properly trained to use equipment, or a general reluctance to wear safety glasses and lab coats. But, just as bottles of unidentified solvents can be stashed guiltily in the depths of a fume cupboard, so such problems are often pushed to the back of the mind, and only properly confronted after an accident.

Most worrying is that it seems researchers only change their attitudes to safety when affected directly by an accident, such as in their own laboratory. A tragedy elsewhere is not always sufficient motivation. After the 2009 death of research assistant Sheharbano Sangji following a fire in the chemistry department at the University of California, Los Angeles (UCLA), safety policies there, such as snap lab inspections to make sure researchers wear protective coats, were reviewed and tightened. But the impact outside the University of California system is hard to determine, and there is little evidence that other chemistry laboratories have responded by changing their practices (see page 270). This could soon change - the deaths of both Sangii and Dufault will feed into ongoing federal-level inquiries into laboratory safety by the US Chemical Safety and Hazard Investigation Board and the National Research Council.

It is certainly true that laboratory researchers work in much safer conditions now than during the more reckless days of the 1950s and 60 s. But such improvements must not breed complacency. For years, environmental health and safety officers have complained that there is no good source of consistent data on laboratory accidents, which could be studied to determine effective safety interventions. As such, it is impossible to say with certainty which laboratories perform well on safety and which badly. And no one can rigorously compare academia's accident rate to that of industry.

UCLA has just announced a centre to study laboratory safety, which may start to pull together some of this information. That would be a good start, and many of the data it would need for the task already exist. Individual research departments in both academia and industry often keep statistics far more detailed than required by federal authorities recording minor incidents and near misses, as well as major accidents. The American Chemical Society's health and safety division has started informally tracking statistics of deaths in academia and industry, to see if any discernible patterns arise. Last year, it surveyed the safety culture in chemistry laboratories, and it plans to repeat the exercise.
The UCLA centre would be a good place to pool this knowledge and make it widely available - and not just between health and safety officers, who already discuss experiences and data. There are useful examples of collaboration on safety issues elsewhere, such as an MIT peer-review process with the National University of Singapore, in which each institution audited the health and safety programmes of the other. If asked, researchers are usually more than happy to make safety policies available or send them out to others. Imperial College London and UCLA both
"Poor safety practices are often pushed to the back of the mind and only confronted after an accident." make their safety plans widely available and grant requests for information from researchers in other countries.

To see safety precautions as a drag on research is an irresponsible and counterproductive attitude, but one that is hard to change. At UCLA, for example, too many researchers see newly introduced safety officers as 'police' to skirt round, rather than experts with whom to collaborate.

Leaders of research projects must take responsibility for the safety of the scientists doing the work, and must start to work with safety officers, rather than endure them. In turn, senior figures in academic departments must realize that practices and priorities have changed since their earlier days, and be willing to shut down laboratories until any potentially dangerous working practices are improved.

The circumstances that ended the life of Michele Dufault last week may have been unusual, even unique. But universities and researchers who feel that there are no lessons to learn from such accidents are a danger to themselves and others.

\section{Fix the PhD}

\section{No longer a guaranteed ticket to an academic career, the PhD system needs a serious rethink.}

$\mathrm{T}$ The world has many problems and it will take a lot of bright, educated people to solve them. So, on the face of it, it seems like a good thing that more and more people are earning $\mathrm{PhDs}$ in science, technology and engineering. Most countries, convinced that higher education and scientific research are key to economic growth and prosperity, are expanding doctoral education in science. The thought, as one researcher who has studied doctoral-education trends puts it, is that you can "grow PhDs like mushrooms".

The consequence of that mushrooming depends on where it is taking place, and in which discipline, as our overview of $\mathrm{PhD}$ systems 